\title{
A Space-Time Network for Telecommuting versus Commuting Decision-Making
}

\author{
Anna Nagurney* \\ Department of Finance and Operations Management \\ Isenberg School of Management \\ University of Massachusetts \\ Amherst, Massachusetts 01003 \\ June Dong \\ School of Business \\ State University of New York at Oswego \\ Oswego, New York 13126 \\ Patricia L. Mokhtarian \\ Department of Civil and Environmental Engineering \\ University of California \\ Davis, California 95616
}

November 2000; revised May 2001; December 2001

Papers in Regional Science 82(4): (2003), pp 451-473

\begin{abstract}
In this paper, we develop a theoretical framework for the study of telecommuting versus commuting decision-making over a fixed time horizon, such as a work week through the use of a space-time network to conceptualize the decision-makers' choices over space and time. The decision-makers are multiclass and multicriteria ones and perceive the criteria of travel cost, travel time, and opportunity cost in an individual fashion. The model is a network equilibrium one and allows for the prediction of the equilibrium flows and, hence, the number of periods that members of each class of decision-makers will telecommute or commute. Qualitative properties of the equilibrium are obtained and an algorithm is given, along with convergence results, and applied to numerical examples.
\end{abstract}

JEL classification: C61, L86, L91, L96, R41

Key words: Transportation and telecommunication networks, telecommuting and commuting, space-time networks, variational inequalities 
* Corresponding author; e-mail: nagurney@gbfin.umass.edu; fax no: 413-545-3858 


\section{Introduction}

The topic of transportation and its relationships to telecommunications has been a subject of research interest for close to forty years (cf. Memmott (1963), Jones (1973), Khan (1976), Nilles, at el. (1976), Albertson (1977), and Harkness (1977)). Commuting, in particular, as one of the most common uses of transportation, and, telecommuting, made possible by the advent of technologies, have garnered special attention. Indeed, telecommuting has been explored in many studies in terms of its potential impact on reducing the negative effects of transportation such as congestion and environmental degradation due to pollution. For conceptual studies on this topic, see Salomon (1986), and Mokhtarian (1990, 1998); for empirical studies, see Nilles (1988), Mokhtarian (1991), and Mokhtarian, Handy, and Salomon (1995).

Recently, Nagurney, Dong, and Mokhtarian (2000) proposed an integrated multiclass, multicriteria network equilibrium framework for telecommuting versus commuting (see, also, Nagurney, Dong, and Mokhtarian (2001)). In particular, they demonstrated that, through the use of appropriate criteria, and the extension of the concept of a network to include not only links associated with physical transportation but also links associated with telecommunications and, hence, virtual transportation, one could predict the number of decision-makers of each class that would telecommute versus commute. Importantly, they allowed each class of decision-maker to weight the criteria of travel time, travel cost, and opportunity cost in an individual fashion.

In this paper, in contrast, we address the more general question as to how many days (given, say, a weekly horizon), one can expect classes of individuals to telecommute or to commute. In Nagurney, Dong, and Mokhtarian (2000), on the other hand, the focus was essentially on one time period with no allowance made for decision-making over time. That approach, nevertheless, was an equilibrium approach, as is the one proposed in this paper. Moreover, that paper presented elastic demand models which had locational implications as well, whereas here we focus on fixed demand models and not only on the question of whether or not to telecommute (versus commute) but how frequently this choice is made.

The crucial concept that we utilize in this paper is that of a space-time network in order to abstract the decision-making not only over space, but also over time, where time here 
is considered to be a finite horizon of periods, such as a work week consisting of five days. The framework allows one to predict how many decision-makers of each class will choose to telecommute on any given day of the week. The functions representing the criteria on the links of the network capture the dependence of the criteria on the flows over both space and over time. For example, there may be individuals of a class who select to telecommute five days of the work week, whereas others may choose to telecommute only one or two days.

The use of space-time networks was also made by Nagurney and Aronson (1989) and by Nagurney and Kim (1991) in order to formulate and study multiperiod spatial price equilibrium problems originated by Takayama and Judge (1971).

Given recent legislation that permits federal employees to select the telecommuting option, as well as a resurgence of interest on this topic (see Hafner (2000)), a theoretical framework that can model telecommuting versus commuting behavior over a time horizon is clearly also of practical relevance. The number of telecommuters in the USA (see Glater (2001)) has risen in the past decade from 4 million to 23.6 million. However, how often individuals choose to telecommute versus commute (and, typically, on what days) is still an open question both theoretically and empirically. Some recent summary survey results concerning telecommuting intensity can be found in International Telework Association \& Council (2000). See also Shore (2000) for an overview of teleworking, which highlights that different workers may choose to telecommute a different number of days.

In this paper, we hope to contribute, both conceptually and theoretically, to the understanding of telecommuting versus commuting decision-making not only in a spatial context but also in a time dimension. This is the first attempt at such a formalization.

We note that multicriteria traffic network models were introduced by Quandt (1967) and Schneider (1968) and further developed by Dial (1979) who proposed an uncongested model and Dafermos (1981) who introduced congestion and derived an infinite-dimensional variational inequality formulation. Subsequent contributions were made by Leurent (1993, 1996, 1998), Marcotte, Nguyen, and Tanguay (1996), Marcotte (1998), Dial (1999), and Nagurney and Dong (2000). A thorough discussion as to the particulars of the contributions can be found in Nagurney and Dong (2000). The former authors, however, considered two criteria, whereas in this paper, as in the paper of Nagurney, Dong, and Mokhtarian (2000), we 
consider three criteria, with the addition of an opportunity cost to travel time and travel cost. Moreover, the preceding authors did not focus on telecommuting as an option. Furthermore, in contrast to the previous authors, we allow the weights associated with the criteria to be not only class but also link-dependent. Hence, certain classes of decision-makers may associate a higher or a lower weight with a criterion depending on the period or day and/or whether the link is a telecommuting link or not.

This paper is organized as follows. In Section 2, we develop the multiclass, multicriteria network equilibrium model and present the space-time network which allows the conceptualization of the problem. On such a network, a path corresponds to decision-making over different time periods. Here, for definiteness, we focus on a time period being a day. However, the framework is more general and one need not limit oneself thus. Indeed, a period could correspond to a week with the overall time horizon under study then corresponding, say, to a month. The governing equilibrium conditions are derived and two equivalent variational inequalities in path flows and in link loads, respectively, presented.

In Section 3, we present some qualitative properties of the equilibrium pattern, as well as obtain conditions on the function that enters the variational inequality problem which allow us to establish convergence of the algorithmic scheme in Section 4.

In Section 5, we apply the algorithm to several numerical examples for illustrative purposes. Finally, in Section 6, we summarize the results and present our conclusions. 


\section{The Model}

In this section, we develop the multiperiod, multiclass, multicriteria network equilibrium model for telecommuting versus commuting. We let $\mathcal{T}$ denote the finite-time horizon with, typically, $\mathcal{T}$ being set equal to 5 working days of the week, and use the index $\tau=1,2, \ldots, \mathcal{T}$, to denote the time period or day.

\section{The Space-Time Network}

We assume that there are $n$ locations with a subset of the locations corresponding to residential locations, employment locations, teleworking centers, as well as intermediate locations for transportation (or telecommunications) purposes, respectively. The space-time network will consist of $\mathcal{T}$ subnetworks with each subnetwork $\tau$ corresponding to the choices available within time period $\tau$. We index the locations for a subnetwork $\tau$ of the space-time network corresponding to time period $\tau$ as follows: $(1, \tau), \ldots,(n, \tau)$; with $\tau$ ranging from 1 through $\mathcal{T}$. We assume that the number of locations within each subnetwork is fixed at $n$, which is also the number of nodes in each subnetwork. Hence, the total number of nodes in the space-time network will be equal to $\mathcal{T} n$.

We now discuss the links on the space-time network. The links will be links on each subnetwork plus connecting links which join two successive subnetworks. A link in our framework can represent either a physical link corresponding to a classical transportation link or a virtual link corresponding to a telecommunication link which decision-makers can select as a telecommuting option. For example, in Figure 1, we present a conceptualization for period $\tau$ of a telecommuting versus a commuting problem, which corresponds to subnetwork $\tau$. In Figure 1, nodes $(1, \tau)$ and $(2, \tau)$ depict the residential locations in time period (day) $\tau$; node $(6, \tau)$ denotes the employment location, and nodes $(3, \tau)$ and $(4, \tau)$ denote work center locations from which the decision-makers can telecommute. Hence, links: $((1, \tau),(6, \tau))$; $((2, \tau),(6, \tau)) ;((3, \tau),(6, \tau))$, and $((4, \tau),(6, \tau))$ represent telecommuting links with all other links in Figure 1 being transportation links.

A sequence of links from a residential location to an employment location within a time period is termed a "route" and is denoted by $r$. Note that, as in Dial (1996), a route can represent a mode of transportation in this context (for example, public or private). 


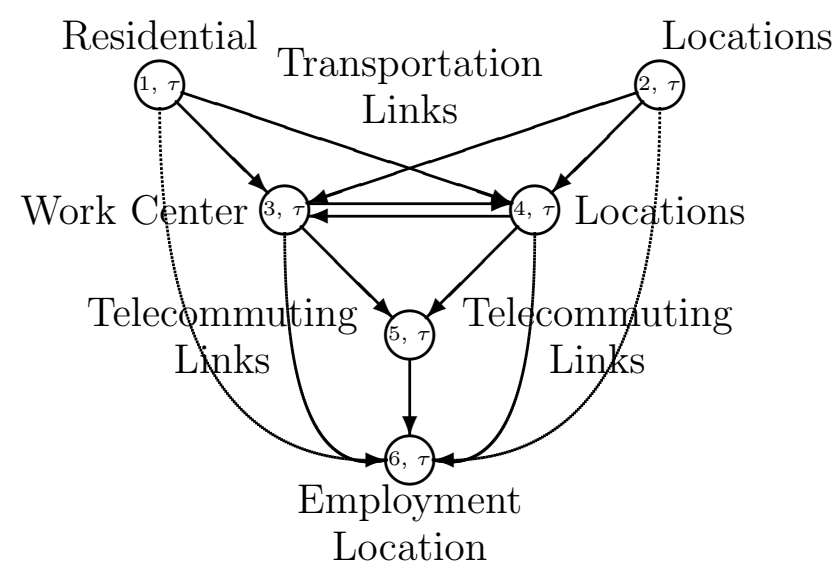

Figure 1: The subnetwork for the conceptualization of commuting versus telecommuting within time period $\tau$

Furthermore, in our framework, since a mode of transportation includes telecommunications, a route can also represent a mode of telecommuting.

A space-time network for a time horizon $\mathcal{T}$, hence, consists of $\mathcal{T}$ copies of a subnetwork $\tau$ with $\tau$ ranging from 1 through $\mathcal{T}$ to denote the subnetworks plus additional links to connect the subnetwork within a time period with the subsequent subnetwork. Thus, from each employment location node in time period $\tau$ we construct a link joining the appropriate residential location node in time period $\tau+1$, where the residential and employment nodes have been identified as the locations where individuals live and work at, respectively. We refer the reader to Figure 2 for the space-time representation of the example given in Figure 1. 
We now define a path in the space-time network. A path is used to represent decisions over space and time and consists of a sequence of links (assumed acyclic) from a residential location node in period 1 to an employment location node in time period $\mathcal{T}$. A residential location node in time period 1 is termed, henceforth, an origin node and the employment location node in time period $\mathcal{T}$ is a destination node with such a pair of nodes referred to as an origin/destination (O/D) pair. A path, thus, consists of a sequence of routes, which a decision-maker selects joined by the "connecting" links between the successive subnetworks. For example, cf. Figure 2, a decision-maker who selects the path consisting of the links: $(((1,1),(6,1)) ;((6,1),(1,2)) ;((1,2),(6,2)) ; \ldots,((6,4),(1,5)) ;((1,5),(6,5)))$ will telecommute each of the five days; other sequences of links represent other possible options.

Note that a path corresponding to a particular O/D pair must be constructed so that the appropriate residential location/employment location pair of nodes is included for each subnetwork. Otherwise, it would mean that one might switch within the time horizon either one's residential location, or one's employment location, or both. To fix this idea, we present a very simple example in Figure 3 in which there are 3 residential locations and only a single employment location. Consider now the origin/destination pair of nodes $((1,1),(4,5))$ and note that there is only one path that connects this $\mathrm{O} / \mathrm{D}$ pair and that satisfies the above restriction. It is drawn in bold-face in Figure 3.

The space-time network, hence, is a general network $G=[\mathcal{N}, \mathcal{L}]$, (but of special, multiperiod structure), where $\mathcal{N}$ denotes the set of nodes in the network and $\mathcal{L}$ the set of directed links. Let now $a$ denote a link of the network connecting a pair of nodes and let $p$ denote a path, consisting of a sequence of links connecting an origin/destination (O/D) pair of nodes. There are $N$ links in the space-time network and $N_{P}$ paths. Let $\Omega$ denote the set of $J$ $\mathrm{O} / \mathrm{D}$ pairs. The set of paths connecting the $\mathrm{O} / \mathrm{D}$ pair $\omega$ is denoted by $P_{\omega}$ and the entire set of paths in the network by $P$. For example, if there are $N_{r}$ routes connecting a residential/employment location pair of nodes, then there will be $N_{r}{ }^{\mathcal{T}}$ paths for the corresponding $\mathrm{O} / \mathrm{D}$ pair, which recall consists of the residential location node in time period 1 and the employment location node in time period $\mathcal{T}$. 


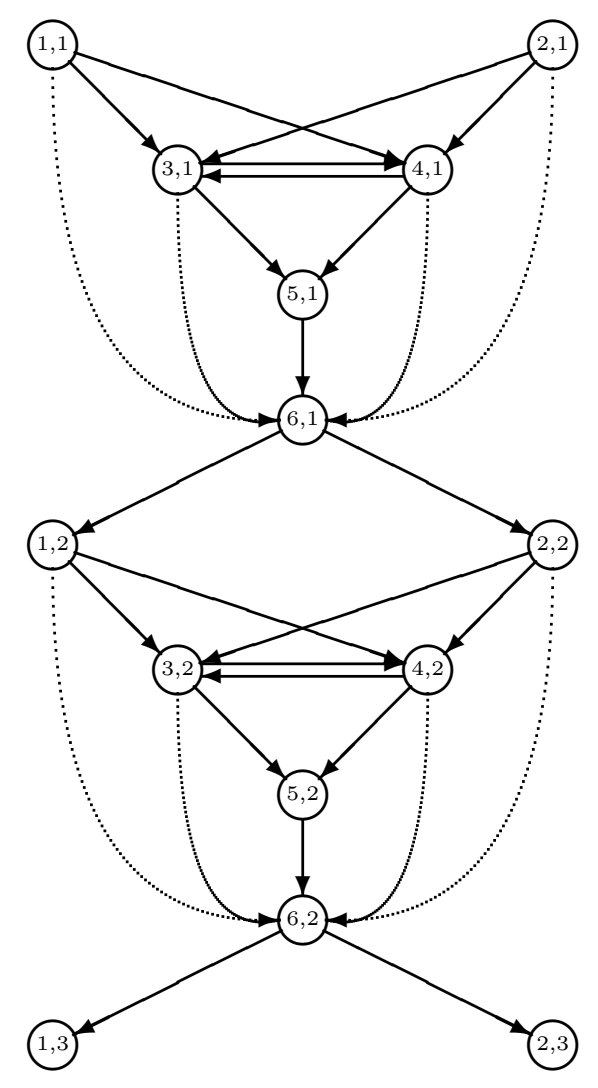

Day 1

Day 2

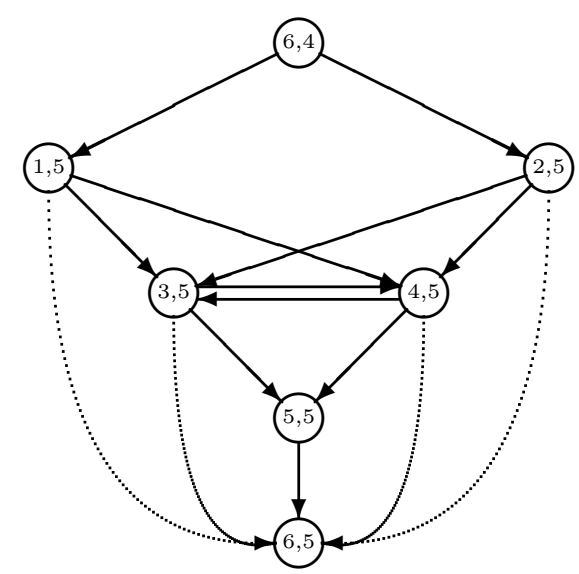

Day 5

Figure 2: A space-time network for an example with a 5 day time horizon 


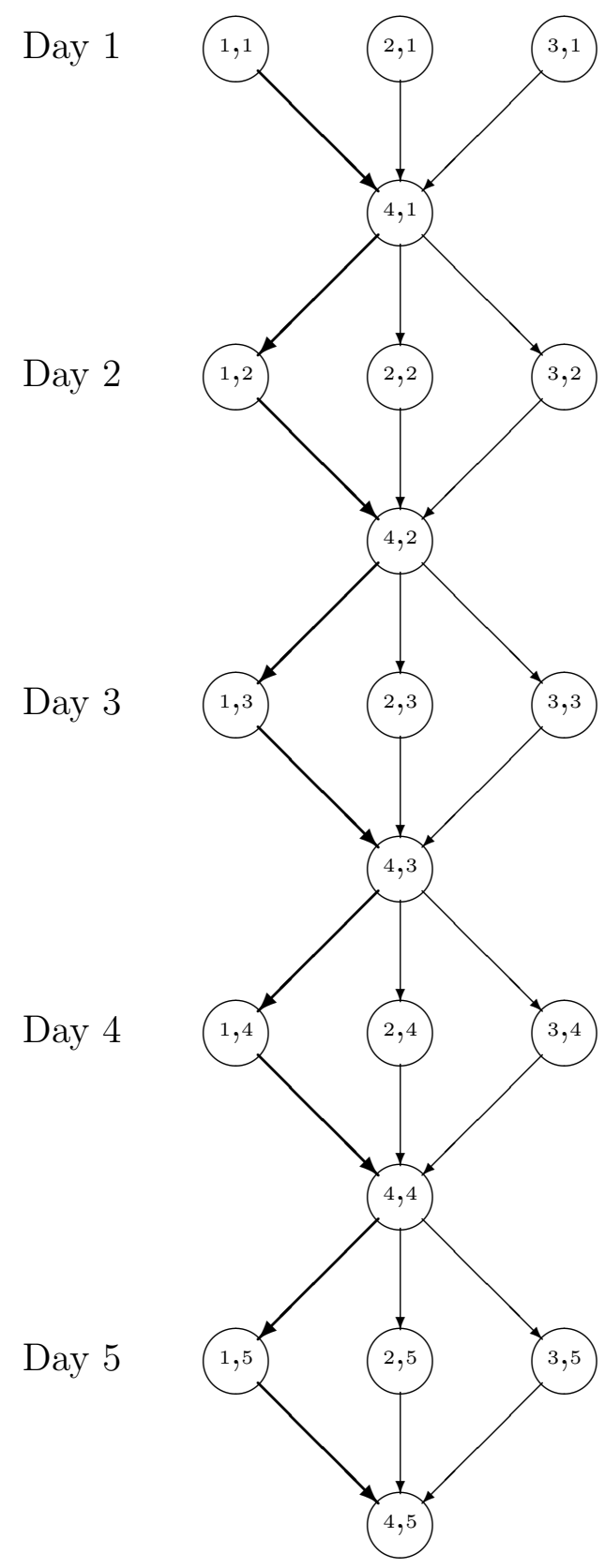

Figure 3: Example of the construction of a path 


\section{The Conservation of Flow Equations}

Assume that there are $k$ classes of decision-makers in the network with a typical class denoted by $i$. Let $f_{a}^{i}$ denote the flow of class $i$ on link $a$ and let $x_{p}^{i}$ denote the nonnegative flow of class $i$ on path $p$. The relationship between the link flows by class and the path flows is:

$$
f_{a}^{i}=\sum_{p \in P} x_{p}^{i} \delta_{a p}, \quad \forall i, \quad \forall a \in \mathcal{L}
$$

where $\delta_{a p}=1$, if link $a$ is contained in path $p$, and 0 , otherwise. Hence, the flow of a class of decision-maker on a link is equal to the sum of the flows of the class on the paths that contain that link.

In addition, let $f_{a}$ denote the total flow on link $a$, where

$$
f_{a}=\sum_{i=1}^{k} f_{a}^{i}, \quad \forall a \in \mathcal{L} .
$$

Therefore, the total flow on a link is equal to the sum of the loads of all classes on that link. Group the class link flows into the $k N$-dimensional column vector $\tilde{f}$ with components: $\left\{f_{a}^{1}, \ldots, f_{N}^{1}, \ldots, f_{a}^{k}, \ldots, f_{N}^{k}\right\}$ and the total link flows: $\left\{f_{a}, \ldots, f_{N}\right\}$ into the $N$-dimensional column vector $f$. Also, group the class path flows into the $k N_{P}$-dimensional column vector $\tilde{x}$ with components: $\left\{x_{p_{1}}^{1}, \ldots, x_{p_{N_{P}}}^{k}\right\}$. The travel demand associated with origin/destination (O/D) pair $\omega$ and class $i$ will be denoted by $d_{\omega}^{i}$. We group the travel demands into a column vector $d \in R^{k J}$. Clearly, the travel demands must satisfy the following conservation of flow equations:

$$
d_{\omega}^{i}=\sum_{p \in P_{\omega}} x_{p}^{i}, \quad \forall i, \forall \omega
$$

Hence, we assume here that the demand is fixed and known. In Nagurney, Dong, and Mokhtarian (2000) the single period, elastic demand analogue of this problem was formulated; see also Nagurney, Dong, and Mokhtarian (2001).

\section{The Generalized Cost Structure}

Note that there may be as many classes of decision-makers on this network as there are groups who perceive the tradeoffs among travel cost, travel time, and opportunity cost in a 
similar fashion.

We are now ready to describe the functions associated with the links. We emphasize that because of the structure of the space-time network we allow the functions on the links to depend, in general, upon the flow on any link in the space-time network and, consequently, we can capture dependence over time since flows on distinct subnetworks correspond to flows in distinct time periods.

We assume, as given, a travel time function $t_{a}$ associated with each link $a$ in the network, where

$$
t_{a}=t_{a}(f), \quad \forall a \in \mathcal{L},
$$

where (4) represents the time that it takes to traverse link $a$, and a travel cost function $c_{a}$ associated with each link $a$, that is,

$$
c_{a}=c_{a}(f), \quad \forall a \in \mathcal{L},
$$

with both these functions assumed to be continuous. Note that here we allow for the general situation in which both the travel time and the travel cost can depend on the entire link flow pattern. Nagurney, Dong, and Mokhtarian (2000) also considered such general functions but did not relate them to distinct time periods.

In addition, in order to capture the opportunity costs associated with commuting versus telecommuting tradeoffs, we also introduce an opportunity cost $o_{a}$ associated with each link in the network, where

$$
o_{a}=o_{a}(f), \quad \forall a \in \mathcal{L} .
$$

For example, as discussed in Nagurney, Dong, and Mokhtarian (2000), we can expect the opportunity cost associated with a telecommunication link to be higher than that of a transportation link since telecommuters do not have the opportunity of socializing and discussing projects face to face with colleagues, among other factors.

Note that, in general, one need not associate the above functions with the connecting links. However, we do not exclude the possibility which may enhance modeling flexibility.

We assume that each class of decision-maker $i$ has his own perception of the trade-offs among travel time, travel cost, and opportunity cost associated with each link $a$, which are 
represented, respectively, by the nonnegative weights $w_{1 a}^{i}, w_{2 a}^{i}$, and $w_{3 a}^{i}$. Here $w_{1 a}^{i}$ denotes the weight associated with class $i$ 's travel time on link $a, w_{2 a}^{i}$ denotes the weight associated with class $i$ 's travel cost on $\operatorname{link} a$, and $w_{3 a}^{i}$ denotes the weight associated with class $i$ 's opportunity cost on link $a$. The weights $w_{1 a}^{i}, w_{2 a}^{i}$, and $w_{3 a}^{i}$ are link-dependent and, hence, can incorporate such link-dependent factors as safety, comfort, view, and sociability factors.

Link-dependent weights were proposed in Nagurney and Dong (2000) and provide a greater level of generality and flexibility in modeling decision-making on networks than weights that are identical for the travel time and for the travel cost on all links for a given class (see Nagurney (2000), Dafermos (1981), and the references therein). Note that in order to model telecommuting versus commuting some links may only have opportunity costs associated with them whereas others may not even include opportunity costs (or have zero associated weights). Moreover, due to the structure of the space-time network one may have an opportunity cost in a specific time period depend on the flows of telecommuters in preceding and subsequent time periods. Thus, one can incorporate the costs associated with telecommuting in several time periods.

The generalized cost function of class $i$ associated with link $a$, denoted by $u_{a}^{i}$, is defined as:

$$
u_{a}^{i}=w_{1 a}^{i} t_{a}+w_{2 a}^{i} c_{a}+w_{3 a}^{i} o_{a}, \quad \forall i, \quad \forall a \in \mathcal{L}
$$

In other words, the generalized cost on a link as experienced by a class of decision-maker is a weighting of the travel cost, travel time, and opportunity cost on that link, which represent the criteria used in decision-making.

In view of $(2)-(7)$, we may write

$$
u_{a}^{i}=u_{a}^{i}(\tilde{f}), \quad \forall i, \quad \forall a \in \mathcal{L},
$$

and group the generalized link costs into the $k N$-dimensional row vector $u$ with components: $\left\{u_{a}^{1}, \ldots, u_{N}^{1}, \ldots, u_{a}^{k}, \ldots, u_{N}^{k}\right\}$.

Let $v_{p}^{i}$ denote the generalized cost of class $i$ associated with traversing path $p$, where

$$
v_{p}^{i}=\sum_{a \in \mathcal{L}} u_{a}^{i}(\tilde{f}) \delta_{a p}, \quad \forall i, \forall p
$$


Hence, the generalized cost, as perceived by a class, associated with traversing a path is its weighting of the travel times, the travel costs, and the opportunity costs on links which comprise the path, where the links in each subnetwork represent the possible choices available to the decision-maker within that time-period. We group the generalized path costs into the column vector $v \in R^{k N_{P}}$.

\section{The Behavioral Assumption}

The behavioral assumption that we utilize here has also been used by Nagurney (2000) and Nagurney, Dong, and Mokhtarian (2000) and is based on the behavioral assumption underlying traffic network equilibrium problems, assuming user-optimizing behavior (see Beckmann, McGuire, and Winsten (1956), Dafermos and Sparrow (1969)), in that we assume that each class of decision-maker in the space-time network selects (subject to constraints) his "travel" path so as to minimize the generalized cost on the path, given that all other decision-makers have made their choices. Note that paths in the space-time framework correspond to decisions not only over space but also over time. Hence, the use of this behavioral assumption is reasonable and classical, in a sense, but, at the same time, novel.

In particular, we have the following network equilibrium conditions for the problem outlined above:

\section{Network Equilibrium Conditions}

For each class $i$, for all $\mathrm{O} / \mathrm{D}$ pairs $\omega \in \Omega$, and for all paths $p \in P_{\omega}$, the flow $\tilde{x}^{*}$ is said to be in equilibrium if the following condition holds:

$$
v_{p}^{i}\left(\tilde{x}^{*}\right)\left\{\begin{array}{lll}
=\lambda_{\omega}^{i}, & \text { if } & x_{p}^{i *}>0 \\
\geq \lambda_{\omega}^{i}, & \text { if } & x_{p}^{i^{*}}=0 .
\end{array}\right.
$$

In other words, all utilized paths by a class connecting an O/D pair have equal and minimal generalized costs and these costs are equal to the disutility $\lambda_{\omega}^{i}$ associated with the class and $\mathrm{O} / \mathrm{D}$ pair.

We now provide two alternative, but, equivalent, variational inequality formulations of the equilibrium conditions (10) in path flows, and in link flows, respectively. The path flow formulation will be utilized in the construction of the tatonnement process in Section 3 . The 
link flow formulation, on the other hand, will be utilized in Section 4 to obtain properties needed for the convergence of the algorithmic scheme in Section 5.

Specifically, in light of Theorem 1 in Nagurney (2000), we can write down immediately the variational inequality formulations below.

\section{Theorem 1: Variational Inequality Formulations}

A multicriteria, multiclass path flow pattern $\tilde{x}^{*} \in \mathcal{K}^{1}$ is a network equilibrium, that is, satisfies equilibrium conditions (10), if and only if it satisfies the variational inequality problem:

\section{Path Flow Formulation:}

$$
\sum_{i=1}^{k} \sum_{\omega \in \Omega} \sum_{p \in P_{\omega}} v_{p}^{i}\left(\tilde{x}^{*}\right) \times\left(x_{p}^{i}-x_{p}^{i *}\right) \geq 0, \quad \forall \tilde{x} \in \mathcal{K}^{1},
$$

where $\mathcal{K}^{1} \equiv\{\tilde{x} \mid \tilde{x} \geq 0$, and satisfies $(3)\}$, or, in standard variational inequality form (cf. Nagurney (1999)):

$$
\left\langle F\left(X^{*}\right)^{T}, X-X^{*}\right\rangle \geq 0, \quad \forall X \in \mathcal{K},
$$

where $F \equiv v, X \equiv \tilde{x}$, and $\mathcal{K} \equiv \mathcal{K}^{1}$, and $\langle\cdot, \cdot\rangle$ denotes the inner product in $k N_{P}$-dimensional space, or, equivalently, $\tilde{f}^{*} \in \mathcal{K}^{2}$ is an equilibrium link flow pattern if and only if it satisfies the variational inequality problem:

\section{Link Flow Formulation:}

$$
\left\langle u\left(\tilde{f}^{*}\right)^{T}, \tilde{f}-\tilde{f}^{*}\right\rangle \geq 0, \quad \forall \tilde{f} \in \mathcal{K}^{2},
$$

where $\mathcal{K}^{2} \equiv\{\tilde{f} \mid \exists \tilde{x} \geq 0$, and satisfying $(1),(2),(3)\}$, or, in standard variational inequality form:

$$
\left\langle F\left(X^{*}\right)^{T}, X-X^{*}\right\rangle \geq 0, \quad \forall X \in \mathcal{K},
$$

where $F \equiv u, X \equiv \tilde{f}, \mathcal{K} \equiv \mathcal{K}^{2}$, and $\langle\cdot, \cdot\rangle$ denotes the inner product in $k N$-dimensional Euclidean space.

Proof: Follows from the proof of Theorem 1 in Nagurney (2000).

If there is only a single time period, then the model collapses to the model of Nagurney, Dong, and Mokhtarian (2000), in the case of fixed demands. 


\section{Qualitative Properties}

We now derive some qualitative properties of the solutions to variational inequalities (11a) and (12a). Note that the feasible set $\mathcal{K}^{1}$ underlying the variational inequality (11a) is a compact set because of the fixed travel demand as is the feasible set $\mathcal{K}^{2}$ underlying variational inequality (12a). Moreover, the functions that enter the variational inequality problems (11a) and (12a) are continuous. Referring to Nagurney (1999), we can immediately present the following result, which is a standard existence result from the theory of variational inequalities (see also Kinderlehrer and Stampacchia (1980)).

\section{Theorem 2: Existence}

Let $t, c$, and o be given continuous functions. Then both variational inequality (11a) and variational inequality (12a) have at least one solution.

We now turn to examining uniqueness. In particular, we consider a special case of the above model in which we establish uniqueness not of the vector of class link flows $\tilde{f}^{*}$ but, rather, the uniqueness of the total link flows $f^{*}$. For this purpose, we utilize the link flow variational inequality (12a).

Specifically, consider a generalized cost function of the form:

$$
u_{a}^{i}=\psi_{a}^{i} t_{a}+\xi_{a}^{i} c_{a}+\left(1-\psi_{a}^{i}-\xi_{a}^{i}\right) o_{a}, \quad \forall a, i,
$$

where

$$
t_{a}=g_{a}(f)+\alpha_{a}, \quad c_{a}=g_{a}(f)+\beta_{a}, \quad o_{a}=g_{a}(f)+\gamma_{a}, \quad \forall a \in \mathcal{L} .
$$

Hence, the generalized cost function $u_{a}^{i}$ for each class and link is a weighted average of the travel time, the travel cost, and the opportunity cost on a link. Moreover, the variable term is identical for the travel time, the travel cost, and opportunity cost on a given link. Here $\psi_{a}^{i}$ and $\xi_{a}^{i}$ are the link-dependent weights for class $i$ travelers. Assume now that $t, c$, and $o$ are each strictly monotone in $f$, that is,

$$
\begin{aligned}
& \left\langle\left(t\left(f^{1}\right)-t\left(f^{2}\right)\right)^{T}, f^{1}-f^{2}\right\rangle>0, \quad \forall f^{1}, f^{2} \in \mathcal{K}^{2}, \quad f^{1} \neq f^{2}, \\
& \left\langle\left(c\left(f^{1}\right)-c\left(f^{2}\right)\right)^{T}, f^{1}-f^{2}\right\rangle>0, \quad \forall f^{1}, f^{2} \in \mathcal{K}^{2}, \quad f^{1} \neq f^{2},
\end{aligned}
$$


and

$$
\left\langle\left(o\left(f^{1}\right)-o\left(f^{2}\right)\right)^{T}, f^{1}-f^{2}\right\rangle>0, \quad \forall f^{1}, f^{2} \in \mathcal{K}^{2}, \quad f^{1} \neq f^{2} .
$$

Then we have the following:

\section{Theorem 3: Uniqueness of the Total Link Flow Pattern in a Special Case}

The total link flow pattern $f^{*}$ induced by a solution $\tilde{f}^{*}$ to variational inequality (12a) in the case of generalized cost functions $u$ of the form (13) and (14), is guaranteed to be unique if the travel time, the travel cost, and the opportunity cost functions are each strictly monotone increasing in $f$.

\section{Proof:}

Assume that there are two solutions to variational inequality (12a) given by $\tilde{f}^{\prime}$ and $\tilde{f}^{\prime \prime}$. Denote the total link flow patterns induced by these class patterns through (2) by $f^{\prime}$ and $f^{\prime \prime}$, respectively. Then, since $\tilde{f}^{\prime}$ is assumed to be a solution we must have that

$$
\begin{gathered}
\sum_{i=1}^{k} \sum_{a \in \mathcal{L}}\left[\psi_{a}^{i}\left(g_{a}\left(f^{\prime}\right)+\alpha_{a}\right)+\xi_{a}^{i}\left(g_{a}\left(f^{\prime}\right)+\beta_{a}\right)+\left(1-\psi_{a}^{i}-\xi_{a}^{i}\right)\left(g_{a}\left(f^{\prime}\right)+\gamma_{a}\right)\right] \times\left(f_{a}^{i}-f_{a}^{i^{\prime}}\right) \geq 0 \\
\forall f \in \mathcal{K}^{2}
\end{gathered}
$$

Similarly, since $\tilde{f}^{\prime \prime}$ is also assumed to be a solution, we must have that

$$
\begin{gathered}
\sum_{i=1}^{k} \sum_{a \in \mathcal{L}}\left[\psi_{a}^{i}\left(g_{a}\left(f^{\prime \prime}\right)+\alpha_{a}\right)+\xi_{a}^{i}\left(g_{a}\left(f^{\prime \prime}\right)+\beta_{a}\right)+\left(1-\psi_{a}^{i}-\xi_{a}^{i}\right)\left(g_{a}\left(f^{\prime \prime}\right)+\gamma_{a}\right)\right] \times\left(f_{a}^{i}-f_{a}^{i^{\prime \prime}}\right) \geq 0 \\
\forall f \in \mathcal{K}^{2}
\end{gathered}
$$

Let $f=f^{\prime \prime}$ and substitute into (18). Similarly, let $f=f^{\prime}$ and substitute into (19). Adding the two resulting inequalities, after algebraic simplifications, yields

$$
\sum_{a \in \mathcal{L}}\left(g_{a}\left(f^{\prime}\right)-g_{a}\left(f^{\prime \prime}\right)\right) \times\left(f_{a}^{\prime}-f_{a}^{\prime \prime}\right) \leq 0,
$$

which is in contradiction to the assumption that the travel time, the travel cost, and the opportunity cost functions are strictly monotone increasing. Hence, we must have that $f^{\prime}=f^{\prime \prime}$. 
In addition, we now derive the monotonicity property as well as Lipschitz continuity which will be used in establishing convergence of the algorithm in Section 4.

\section{Theorem 4: Monotonicity in a Special Case}

Assume that the generalized cost functions $u$ are as in (13) with the travel time, the travel cost, and the opportunity cost functions differing on a given link only by the fixed cost terms as in (14). Assume also that these functions are monotone increasing in $f$. Then the function that enters the variational inequality problem (12b) governing the multiperiod, multiclass, multicriteria traffic network equilibrium model is monotone.

\section{Proof:}

We need to establish that

$$
\left\langle\left(F\left(X^{1}\right)-F\left(X^{2}\right)\right)^{T}, X^{1}-X^{2}\right\rangle \geq 0, \quad \forall X^{1}, X^{2} \in \mathcal{K}^{2},
$$

where $F, X$, and $\mathcal{K}^{2}$ are as defined following (12b) and with $u$ given by (13) and (14).

We have that

$$
\begin{gathered}
\left\langle\left(F\left(X^{1}\right)-F\left(X^{2}\right)\right)^{T}, X^{1}-X^{2}\right\rangle \\
=\sum_{i=1}^{k} \sum_{a \in \mathcal{L}}\left[\left[\psi_{a}^{i}\left(g_{a}\left(f^{1}\right)+\alpha_{a}\right)+\xi_{a}^{i}\left(g_{a}\left(f^{1}\right)+\beta_{a}\right)+\left(1-\psi_{a}^{i}-\xi_{a}^{i}\right)\left(g_{a}\left(f^{1}\right)+\gamma_{a}\right)\right]\right. \\
\left.-\left[\psi_{a}^{i}\left(g_{a}\left(f^{2}\right)+\alpha_{a}\right)+\xi_{a}^{i}\left(g_{a}\left(f^{2}\right)+\beta_{a}\right)+\left(1-\psi_{a}^{i}-\xi_{a}^{i}\right)\left(g_{a}^{i}\left(f^{2}\right)+\gamma_{a}\right)\right]\right] \times\left[f_{a}^{i^{1}}-f_{a}^{i^{2}}\right] \\
=\sum_{i=1}^{k} \sum_{a \in \mathcal{L}}\left[g_{a}\left(f^{1}\right)-g_{a}\left(f^{2}\right)\right] \times\left[f_{a}^{1}-f_{a}^{2}\right] .
\end{gathered}
$$

But, (22) is greater than or equal to zero, under the assumptions above, and, hence, $F(X)$ is monotone.

\section{Theorem 5: Lipschitz Continuity}

If the generalized cost functions $u$ have bounded first-order derivatives, then the function, $F(X)$, that enters the variational inequality (12b) is Lipschitz continuous, that is, there 
exists a positive constant $L$, such that

$$
\left\|F\left(X^{1}\right)-F\left(X^{2}\right)\right\| \leq L\left\|X^{1}-X^{2}\right\|, \quad \forall X^{1}, X^{2} \in \mathcal{K}^{2}
$$

\section{Proof:}

Denote $F(X)=\left(F_{1}(X), \cdots, F_{N}(X)\right)^{T}$. Since $F_{l}(X): R^{k N} \mapsto R^{1}$ is a smooth function, from the Taylor Theorem, we have that, for any $X^{1}, X^{2} \in \mathcal{K}^{2}$, there exist $\xi_{l} \in R^{k N}, l=$ $1, \cdots, k N$, such that

$$
F_{l}\left(X^{1}\right)-F_{l}\left(X^{2}\right)=\nabla F_{l}\left(\xi_{l}\right)\left(X^{1}-X^{2}\right), \quad l=1, \cdots, k N
$$

Let

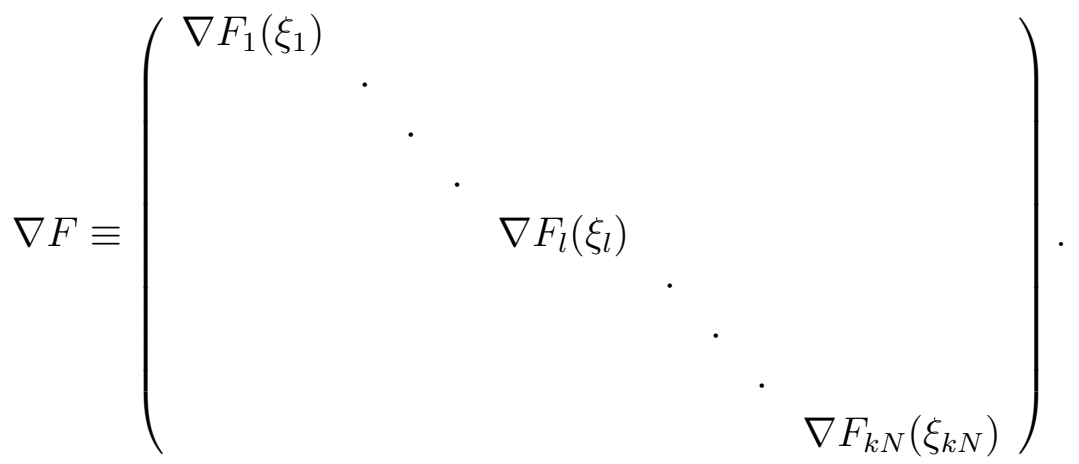

Since the link generalized cost functions have bounded first-order derivatives, there exists an $L \geq 0$, such that

$$
\|\nabla F\| \leq L
$$

Therefore, using (24) and the basic properties of the linear norm operator, we have the following:

$$
\left\|F\left(X^{1}\right)-F\left(X^{2}\right)\right\|=\left\|\left(\begin{array}{c}
\nabla F_{1}\left(\xi_{1}\right)\left(X^{1}-X^{2}\right) \\
\nabla F_{2}\left(\xi_{2}\right)\left(X^{1}-X^{2}\right) \\
\vdots \\
\nabla F_{l}\left(\xi_{l}\right)\left(X^{1}-X^{2}\right) \\
\vdots \\
\nabla F_{k N}\left(\xi_{k N}\right)\left(X^{1}-X^{2}\right)
\end{array}\right)\right\|
$$


In view of (26), one can conclude that

$$
\left\|F\left(X^{1}\right)-F\left(X^{2}\right)\right\| \leq L\left\|X^{1}-X^{2}\right\|, \quad \forall X^{1}, X^{2} \in \mathcal{K}^{2}
$$

Observe that Theorem 4 holds for generalized link cost functions of a specialized form whereas Theorem 6 is not limited to such functions. 


\section{The Algorithm}

In this section, an algorithm is presented which can be applied to solve any variational inequality problem in standard form (see $(12 \mathrm{~b})$ ), that is, Determine $X^{*} \in \mathcal{K}$, satisfying:

$$
\left\langle F\left(X^{*}\right)^{T}, X-X^{*}\right\rangle \geq 0, \quad \forall X \in \mathcal{K} .
$$

The algorithm is guaranteed to converge provided that the function $F$ that enters the variational inequality is monotone and Lipschitz continuous (and that a solution exists). The algorithm is the modified projection method of Korpelevich (1977).

The statement of the modified projection method is as follows, where calk denotes an iteration counter:

\section{Modified Projection Method}

\section{Step 0: Initialization}

Set $X^{0} \in \mathcal{K}$. Let $\mathcal{I}=1$ and let $\gamma$ be a scalar such that $0<\gamma \leq \frac{1}{L}$, where $L$ is the Lipschitz continuity constant (cf. Korpelevich (1977)).

\section{Step 1: Computation}

Compute $\bar{X}^{\mathcal{I}}$ by solving the variational inequality subproblem:

$$
\left\langle\left(\bar{X}^{\mathcal{I}}+\gamma F\left(X^{\mathcal{I}-1}\right)^{T}-X^{\mathcal{I}-1}\right)^{T}, X-\bar{X}^{\mathcal{I}}\right\rangle \geq 0, \quad \forall X \in \mathcal{K} .
$$

\section{Step 2: Adaptation}

Compute $X^{\mathcal{I}}$ by solving the variational inequality subproblem:

$$
\left\langle\left(X^{\mathcal{I}}+\gamma F\left(\bar{X}^{\mathcal{I}}\right)^{T}-X^{\mathcal{I}-1}\right)^{T}, X-X^{\mathcal{I}}\right\rangle \geq 0, \quad \forall X \in \mathcal{K} .
$$

\section{Step 3: Convergence Verification}

If $\max \left|X_{l}^{\mathcal{I}}-X_{l}^{\mathcal{I}-1}\right| \leq \epsilon$, for all $l$, with $\epsilon>0$, a prespecified tolerance, then stop; else, set $\mathcal{I}=: \mathcal{I}+1$, and go to Step 1 . 
We now give an explicit statement of the modified projection method for the solution of variational inequality problem (12a) for the multiperiod, multiclass, multicriteria network equilibrium model.

\section{Modified Projection Method for the Solution of Variational Inequality (12a)}

\section{Step 0: Initialization}

Set $\tilde{f}^{0} \in \mathcal{K}^{2}$. Let $\mathcal{I}=1$ and set $\gamma$ such that $0<\gamma \leq \frac{1}{L}$, where $L$ is the Lipschitz constant for the problem.

\section{Step 1: Computation}

Compute $\overline{\tilde{f}} \mathcal{I} \in \mathcal{K}^{2}$ by solving the variational inequality subproblem:

$$
\sum_{i=1}^{k} \sum_{a \in \mathcal{L}}\left(\bar{f}_{a}^{i}{ }^{\mathcal{I}}+\gamma\left(u_{a}^{i}\left(\tilde{f}^{\mathcal{I}-1}\right)-f_{a}^{i^{\mathcal{I}-1}}\right) \times\left(f_{a}^{i}-\bar{f}_{a}^{i}{ }^{\mathcal{I}}\right) \geq 0, \quad \forall \tilde{f} \in \mathcal{K}^{2}\right.
$$

\section{Step 2: Adaptation}

Compute $\tilde{f}^{\mathcal{I}} \in \mathcal{K}^{2}$ by solving the variational inequality subproblem:

$$
\sum_{i=1}^{k} \sum_{a \in \mathcal{L}}\left(f_{a}^{i^{\mathcal{I}}}+\gamma\left(u_{a}^{i}\left(\overline{\tilde{f}}^{\mathcal{I}}\right)-f_{a}^{i^{\mathcal{I}-1}}\right) \times\left(f_{a}^{i}-f_{a}^{i \mathcal{I}}\right) \geq 0, \quad \forall \tilde{f} \in \mathcal{K}^{2} .\right.
$$

\section{Step 3: Convergence Verification}

If $\left|f_{a}^{i \mathcal{I}}-f_{a}^{i \mathcal{I}-1}\right| \leq \epsilon$, for all $i=1, \cdots, k$, and all $a \in \mathcal{L}$, with $\epsilon>0$, a pre-specified tolerance, then stop; otherwise, set $\mathcal{I}:=\mathcal{I}+1$, and go to Step 1 .

We now state the convergence result for the modified projection method for this model. Note that the algorithm may converge even for functions of more general form provided that they are monotone and Lipschitz continuous. Theorem 6 identifies specific functions for which monotonicity can be readily established. 


\section{Theorem 6: Convergence}

Assume that $u$ takes the form of (13) and (14) and is monotone increasing. Also, assume that $u$ has bounded first-order derivatives. Then the modified projection method described above converges to the solution of the variational inequality (12a) (and (12b)).

\section{Proof:}

According to Korpelevich (1977), the modified projection method converges to the solution of the variational inequality problem of the form $(12 \mathrm{~b})$, provided that the function that enters the variational inequality, $F$ is monotone and Lipschitz continuous and that a solution exists. Existence of a solution follows from Theorem 2. Lipschitz continuity, in turn, follows from Theorem 5 under the assumption that the generalized cost functions have bounded first-order derivatives. The conclusion follows. 


\section{Numerical Examples}

In this section, we present six numerical examples for illustrative purposes. In particular, we solve the examples using the modified projection method described in Section 5. For the solution of the variational inequality subproblems (31) and (32) we utilized the equilibration algorithm of Dafermos and Sparrow (1969). Note that the induced variational inequality subproblems are actually separable quadratic programming problems over a feasible set which is a network. The $\gamma$ parameter in the modified projection method was set to .01, except where noted. The convergence criterion was that the absolute value of the flow for each class of decision-maker at two successive iterations was less than or equal to $\epsilon$, with $\epsilon=.0001$. The space-time network for the numerical examples is given in Figure 4 .

\section{Example 1}

The first numerical example is simple but serves to illustrate interesting features. It consists of a single class of decision-maker with a single residential location and a single employment location. The time horizon $\mathcal{T}=5$. Also, we assume that the choices available to the members of the class of decision-maker are expressed simply as whether to telecommute or to commute. The space-time network is given in Figure 4. It consists of ten nodes (since there are two nodes within each of the 5 time periods or days) and 14 links since there are two links for each of the five time periods and 4 connecting links. There are a total of $2^{5}$ or 32 paths connecting the $\mathrm{O} / \mathrm{D}$ pair $\omega=((1,1),(2,5))$, and these are: $p_{1}=(1,3,4,6,7,9,10,12,13), p_{2}=$ $(1,3,4,6,7,9,10,12,14), p_{3}=(1,3,4,6,7,9,11,12,13), p_{4}=(1,3,4,6,7,9,11,12,14), p_{5}=$ $(1,3,4,6,8,9,10,12,13), p_{6}=(1,3,4,6,8,9,10,12,14), p_{7}=(1,3,4,6,8,9,11,12,13), p_{8}=$ $(1,3,4,6,8,9,11,12,14), p_{9}=(1,3,5,6,7,9,10,12,13), p_{10}=(1,3,5,6,7,9,10,12,14), p_{11}=$ $(1,3,5,6,7,9,11,12,13), p_{12}=(1,3,5,6,7,9,11,12,14), p_{13}=(1,3,5,6,8,9,10,12,13), p_{14}=$ $(1,3,5,6,8,9,10,12,14), p_{15}=(1,3,5,6,8,9,11,12,13), p_{16}=(1,3,5,6,8,9,11,12,14), p_{17}=$ $(2,3,4,6,7,9,10,12,13), p_{18}=(2,3,4,6,7,9,10,12,14), p_{19}=(2,3,4,6,7,9,11,12,13), p_{20}=$ $(2,3,4,6,7,9,11,12,14), p_{21}=(2,3,4,6,8,9,10,12,13), p_{22}=(2,3,4,6,8,9,10,12,13), p_{23}=$ $(2,3,4,6,8,9,11,12,13), p_{24}=(2,3,4,6,8,9,11,12,14), p_{25}=(2,3,5,6,7,9,10,12,13), p_{26}=$ $(2,3,5,6,7,9,10,12,14), p_{27}=(2,3,5,6,7,9,11,12,13), p_{28}=(2,3,5,6,7,9,11,12,14), p_{29}=$ $(2,3,5,6,8,9,10,12,13), p_{30}=(2,3,5,6,8,9,10,12,14), p_{31}=(2,3,5,6,8,9,11,12,13), p_{32}=$ $(2,3,5,6,8,9,11,12,14)$. 
Telecommute Commute

Day 1

Day 2

Day 3

Day 4

Day 5
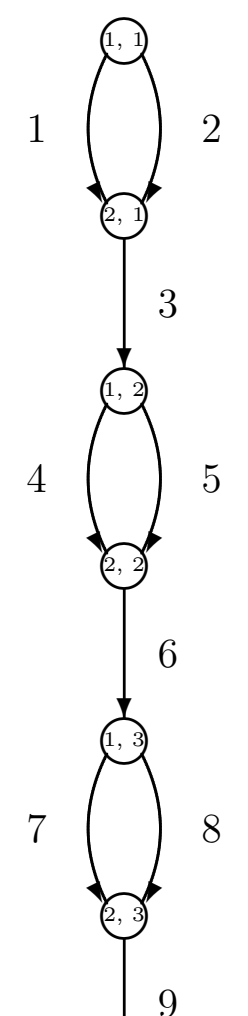

9

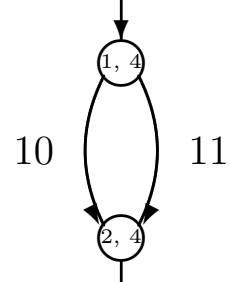

12

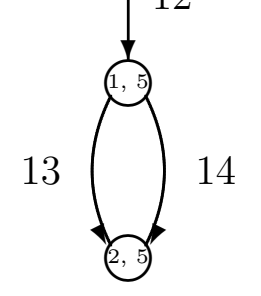

Figure 4: Space-time network for the numerical examples 
Table 1: The Travel Time, Travel Cost, and Opportunity Cost Functions for the Links

\begin{tabular}{|l|l|l|l|}
\hline Link $a$ & $t_{a}(f)$ & $c_{a}(f)$ & $o_{a}(f)$ \\
\hline 1 & $.00005 f_{1}^{4}+.5 f_{1}+.1 f_{2}+.2$ & $.00005 f_{1}^{4}+f_{1}+.5 f_{2}+1$ & $.4 f_{1}+.2 f_{4}+.2$ \\
2 & $.00005 f_{2}^{4}+2 f_{2}+f_{1}+1$ & $.00005 f_{2}^{4}+5 f_{2}+2 f_{1}+2$ & $.2 f_{2}+.1 f_{5}+1$ \\
3 & 0.0000 & 0.0000 & 0.0000 \\
4 & $.00005 f_{4}^{4}+.5 f_{4}+.1 f_{5}+.2$ & $.00005 f_{4}^{4}+f_{4}+.5 f_{5}+1$ & $.3 f_{4}+.2 f_{1}+1$ \\
5 & $.00005 f_{5}^{4}+2 f_{5}+f_{4}+1$ & $.00005 f_{5}^{4}+2 f_{4}+5 f_{5}+2$ & $f_{5}+f_{+} 1$ \\
6 & 0.0000 & 0.0000 & 0.0000 \\
7 & $.00005 f_{7}^{4}+.5 f_{7}+.1 f_{8}+.2$ & $.00005 f_{7}^{4}+f_{7}++.5 f_{8}+1$ & $.5 f_{7}+.1 f_{4}+.2$ \\
8 & $.00005 f_{8}^{4}+2 f_{8}+f_{7}+1$ & $.00005 f_{8}^{4}+5 f_{8}+2 f_{7}+2$ & $2 f_{8}+f_{5}+1$ \\
9 & 0.0000 & 0.0000 & 0.0000 \\
10 & $.00005 f_{10}^{4}+.5 f_{10}+.1 f_{11}+.2$ & $.00005 f_{10}^{4}+f_{10}+.5 f_{11}+1$ & $.5 f_{10}+.1 f_{7}+.2$ \\
11 & $.00005 f_{11}^{4}+2 f_{11}+f_{10}+1$ & $.00005 f_{11}^{4}+5 f_{11}+2 f_{10}+2$ & $f_{11}+.4 f_{8}+1$ \\
12 & 0.0000 & 0.0000 & 0.0000 \\
13 & $.00005 f_{13}^{4}+.5 f_{13}+.1 f_{14}+.2$ & $.00005 f_{13}^{4}+f_{13}+.5 f_{14}+1$ & $.4 f_{13}+.1 f_{10}+.2$ \\
14 & $.00005 f_{14}^{4}+2 f_{14}+f_{13}+1$ & $.00005 f_{14}^{4}+5 f_{14}+2 f_{13}+2$ & $.2 f_{14}+.1 f_{11}+1$ \\
\hline
\end{tabular}

The criteria were as described in Section 2 with the generalized link cost functions constructed according to $(7)$.

The weights were constructed as follows: For class 1, the weights were: $w_{1,1}^{1}=.25$, $w_{2,1}^{1}=.25, w_{3,1}^{1}=1 ., w_{1,2}^{1}=.25, w_{2,2}=.25, w_{3,2}^{1}=1 ., w_{1,3}^{1}=.4, w_{2,3}^{1}=.4, w_{3,3}^{1}=1 .$, $w_{1,4}^{1}=.5, w_{2,4}^{1}=.5, w_{3,4}^{1}=2 ., w_{1,5}^{1}=.4, w_{2,5}^{1}=.5, w_{3,5}^{1}=1 ., w_{1,6}^{1}=.5, w_{2,6}^{1}=.3, w_{3,6}^{1}=2 .$, $w_{1,7}^{1}=.2, w_{2,7}^{1}=.4, w_{3,7}^{1}=1 ., w_{1,8}^{1}=.3, w_{2,8}^{1}=.5, w_{3,8}^{1}=1 ., w_{1,9}^{1}=.6, w_{2,9}^{1}=.2, w_{3,9}^{1}=2 .$, $w_{1,10}^{1}=.3, w_{2,10}^{1}=.4, w_{3,10}^{1}=1 ., w_{1,11}^{1}=.2, w_{2,11}^{1}=.7, w_{3,11}^{1}=1 ., w_{1,12}^{1}=.3, w_{2,12}^{1}=.4$, $w_{3,12}^{1}=1 ., w_{1,13}^{1}=.2, w_{2,13}^{1}=.3, w_{3,13}^{1}=2 ., w_{1,14}^{1}=.5, w_{2,14}^{1}=.2, w_{3,14}^{1}=.1$,

The travel time, travel cost, and opportunity cost functions on the links were as reported in Table 1.

The demand $d_{\omega}^{1}=100$.

The modified projection method converged in 28 iterations. It yielded the following 
equilibrium single class link flow (and total, since there is only 1 class) pattern:

$$
\begin{gathered}
f_{1}^{*}=53.1127, \quad f_{2}^{*}=46.8873, \quad f_{3}^{*}=100.0000, \quad f_{4}^{*}=53.7822, \quad f_{5}^{*}=46.2178, \\
f_{6}^{*}=100.0000, \quad f_{7}^{*}=59.2427, \quad f_{8}^{*}=40.7573, \quad f_{9}^{*}=100.0000, \quad f_{10}^{*}=57.6488, \\
f_{11}^{*}=42.3512, \quad f_{12}^{*}=100.0000, \quad f_{13}^{*}=54.4498, \quad f_{14}^{*}=45.5502,
\end{gathered}
$$

which was induced by the equilibrium single-class path flow pattern:

$$
\begin{aligned}
& x_{p_{1}}^{1 *}=8.8423, \quad x_{p_{2}}^{1 *}=3.1286, \quad x_{p_{3}}^{1 *}=3.2258, \quad x_{p_{4}}{ }^{*}=3.1458, \\
& x_{p_{5}}^{1 *}=3.1435, \quad x_{p_{6}}^{1 *}=3.0630, \quad x_{p_{7}}^{1 *}=1.9958, \quad x_{p_{8}}^{1 *}=3.1984, \\
& x_{p_{9}}^{1 *}=3.1874, \\
& x_{p_{10}}^{1{ }^{*}}=3.6961, \quad x_{p_{11}}^{1 *}=3.2472, \quad x_{p_{12}}^{1 *}=3.1638, \quad x_{p_{13}}{ }^{*}=2.8312, \\
& x_{p_{14}}^{1 *}=2.9486, \quad x_{p_{15}}^{1{ }^{*}}=2.7673, \quad x_{p_{16}}^{1{ }^{*}}=1.580, \quad x_{p_{17}}^{1 *}=3.8039, \\
& x_{p_{18}{ }^{*}}^{1}=3.4827 \\
& x_{p_{19}}^{1}{ }^{*}=3.3014, \quad x_{p_{20}}^{1 *}=3.4188, \quad x_{p_{21}}^{1 *}=3.0862, \quad x_{p_{22}}{ }^{*}=3.0025, \\
& x_{p_{23}}^{1}{ }^{*}=2.5539, \quad x_{p_{24}}^{1 *}=1.3897, \quad x_{p_{25}}^{1{ }^{*}}=3.0516, \quad x_{p_{26}}^{1 *}=4.2542, \\
& x_{p_{27}{ }^{*}}^{1}=3.1870 \text {, } \\
& x_{p_{28}}^{1}{ }^{*}=3.1062, \quad x_{p_{29}}^{1 *}=3.1035, \quad x_{p_{30}}^{1 *}=3.0237, \quad x_{p_{31}}{ }^{*}=3.1221 \text {, } \\
& x_{p_{32}{ }^{*}}^{1 *}=0.0000 \text {. }
\end{aligned}
$$

All the path generalized costs were equal to 1999.4 (approximately to 4 decimal places).

We now discuss the results. Note that only one path, and that is path $p_{32}$, which represents commuting all 5 days of the week was not used. This means that none of the decision-makers in this example opt to commute five days of the week. Path $p_{1}$, on the other hand, represents the option of telecommuting 5 days of the week and only 8.8423 ( see $x_{p_{1}}^{{ }^{*}}$ ) elect this option. This is, nevertheless, under the demand $d_{\omega}=100$, the most popular choice since the largest number of decision-makers make this choice over the 5 day horizon. The next least popular choice (outside of path $p_{32}$ which is not used) is represented by path $p_{16}$ which has the flow 
$x_{p_{16}}^{1}{ }^{*}=1.580$. This represents the following decision: to telecommute on the first day of the week, and to commute on the remaining four days. This may have the interpretation that this class of decision-maker likes to work at home at the beginning of the week (following the weekend, say).

\section{Example 2: Example 1 Subject to an Increase in Demand}

We then proceeded to make the following change. We increased the demand $d_{\omega}$ from 100 to 300 . The modified projection method converged in 5 iterations and yielded the following equilibrium path flow pattern: only path $p_{24}$ was used and it, hence, had all the demand assigned to it, that is, $x_{p_{24}}^{1}{ }^{*}=300$, with all other path flows being, thus, equal to zero. Path $p_{24}$ represents the following decision(s): to commute on days $1,3,4$, and 5 , and to telecommute on day 2. Path $p_{24}$ had a generalized path cost of 1915.2499, and all other paths (which were unused) had (substantially) higher generalized path costs.

\section{Example 3}

In the third numerical example, we added another class of decision-maker, denoted by class 2 , whose weights were as follows: $w_{1,1}^{2}=.5, w_{2,1}^{2}=.3, w_{3,1}^{2}=.1, w_{1,2}^{2}=1 ., w_{2,2}^{2}=1$., $w_{3,2}^{2}=1 ., w_{1,3}^{2}=1 ., w_{2,3}^{2}=1 ., w_{3,3}^{2}=1 ., w_{1,4}^{2}=1$., $w_{2,4}^{2}=1 ., w_{3,4}^{2}=1 ., w_{1,5}^{2}=1 ., w_{2,5}^{2}=1$., $w_{3,5}^{2}=1 ., w_{1,6}^{2}=.5, w_{2,6}^{2}=.5, w_{3,6}^{2}=.5, w_{1,7}^{2}=.5, w_{2,7}^{2}=.4, w_{3,7}^{2}=.4, w_{1,8}^{2}=.4, w_{2,8}^{2}=.3$, $w_{3,8}^{2}=.2, w_{1,9}^{2}=.3, w_{2,9}^{2}=.2, w_{3,9}^{2}=.6, w_{1,10}^{2}=.5, w_{2,10}^{2}=.4, w_{3,10}^{2}=.5, w_{1,11}^{2}=.7$, $w_{2,11}^{2}=.6, w_{3,11}^{2}=.7, w_{1,12}^{2}=.4, w_{2,12}^{2}=.3, w_{3,12}^{2}=.8, w_{1,13}^{2}=.3, w_{2,13}^{2}=.2, w_{3,13}^{2}=.6$, $w_{1,14}^{2}=.2, w_{2,14}^{2}=.3, w_{3,14}^{2}=.9$.

We kept the remainder of the data as in Example 1. We set the demand for class 2 as $d_{\omega}^{2}=100$.

We now present and discuss the results obtained by an application of the modified project method which converged in 7 iterations. Recall that in Example 1, when there was only a single class of decision-maker, then path $p_{32}$ was the only path that was not used by class 1. Now, however, with the introduction of a new class of decision-maker, the following equilibrium pattern was obtained: For class 1 , only path $p_{32}$ was used and, hence, $x_{p_{32}}^{1}{ }^{*}=100$, with an associated generalized path cost given by $v_{p_{32}}^{1}=1200.0499$; all other generalized path 
costs were higher for this class since those paths were not used. Class 2 also only utilized path $p_{32}$ and, hence, the flow for class 2 on that path was $x_{p_{32}}^{2}{ }^{*}=100$. Its generalized cost was $v_{p_{32}}^{2}=1953.9998$, with the other unused paths having higher generalized costs for this class. Interestingly, with the addition of a new class of decision-maker the equilibrium pattern for class 1 changed entirely. Also, interestingly, despite different weights associated with the criteria both decision-makers of class 1 and of class 2 chose to commute 5 days a week!

\section{Example 4: Example 3 Subject to an Increase in Demand for Class 1}

We then made the following perturbation to the data. We increased the demand for class 1 to 300, that is, $d_{\omega}^{1}=300$, but kept the demand for class 2 as in Example 3, that is, $d_{\omega}^{2}=100$. The new equilibrium pattern was computed by the modified projection method in 2 iterations. Now both class 1 and class 2 used solely path $p_{24}$, where recall that this path corresponds to telecommuting on the second day the week and commuting on the remaining 4 days. Interestingly, both classes of decision-makers selected the same option, again.

\section{Example 5: Example 3 Subject to an Increase in Demand for Both Class 1 and Class 2}

We then proceeded to increase the demand for class 2 to 300 , that is, $d_{\omega}^{2}=300$, with the other data as in the example immediately preceding. The modified projection method converged in 4 iterations and the solution stayed the same as in the preceding example.

\section{Example 6: Example 3 Subject to a Decrease in Demand for Both Class 1 and Class 2}

We next decreased the demands for both classes so that $d_{\omega}^{1}=30$ and $d_{\omega}^{2}=30$, and kept all other data as in Example 3. Interestingly, except for 1 path, which was used by both classes, the other paths used were distinct for each class. The modified projection method converged in 243 iterations and yielded the following path flow pattern:

For class 1:

$$
x_{p_{1}}^{1 *}=.3359, \quad x_{p_{2}}^{1 *}=1.9953, \quad x_{p_{9}}^{1 *}=1.4688, \quad x_{p_{10}}^{1 *}=2.7508,
$$




$$
x_{p_{17}}^{1 *}=1.8524, \quad x_{p_{18}}^{1 *}=4.1009, \quad x_{p_{25}}^{1 *}=2.4664, \quad x_{p_{26}}^{1 *}=15.0296,
$$

with all other path flows for this class being equal to zero. The generalized path costs on the used paths was approximately 644.95 for all such paths.

For class 2:

$$
x_{p_{1}}^{2 *}=4.3939, \quad x_{p_{3}}^{2 *}=4.3290, \quad x_{p_{5}}^{2 *}=7.740, \quad x_{p_{7}}^{2 *}=13.5531
$$

with all other path flows for this class being equal to zero. The generalized path costs on the used paths was 809.95, approximately.

Note that both classes used path 1 , that is, there were members of each class who sought to telecommute all five days. However, all other paths used by class 2 were distinct from those chosen by class 1 .

For completeness, we also report the computed equilibrium multiclass link flow pattern and the total link flow pattern:

For class 1:

$$
\begin{gathered}
f_{1}^{1^{*}}=6.5508, \quad f_{2}^{1^{*}}=23.4492, \quad f_{3}^{1^{*}}=30.0000, \quad f_{4}^{1^{*}}=8.2845, \quad f_{5}^{1^{*}}=21.7155 \\
f_{6}^{1^{*}}=30.0000, \quad f_{7}^{1^{*}}=30.0000, \quad f_{8}^{1^{*}}=0.0000, \quad f_{9}^{1^{*}}=30.0000, \quad f_{10}^{1^{*}}=30.0000 \\
f_{11}^{1^{*}}=0.0000, \quad f_{12}^{1^{*}}=30.0000, \quad f_{13}^{1^{*}}=6.1235, \quad f_{14}^{1^{*}}=23.8765
\end{gathered}
$$

For class 2:

$$
\begin{gathered}
f_{1}^{2^{*}}=30.0000, \quad f_{2}^{2^{*}}=0.0000, \quad f_{3}^{2^{*}}=30.0000, \quad f_{4}^{2^{*}}=30.0000, \quad f_{5}^{2^{*}}=0.0000 \\
f_{6}^{2^{*}}=30.0000, \quad f_{7}^{2^{*}}=8.7229, \quad f_{8}^{2^{*}}=21.2771, \quad f_{9}^{2^{*}}=30.0000, \quad f_{10}^{2 *}=12.1179 \\
f_{11}^{2 *}=17.8821, \quad f_{12}^{2 *}=30.0000, \quad f_{13}^{2^{*}}=30.0000, \quad f_{14}^{2 *}=0.0000
\end{gathered}
$$

and

$$
\begin{aligned}
& f_{1}^{*}=36.5508, \quad f_{2}^{*}=23.4492, \quad f_{3}^{*}=60.0000, \quad f_{4}^{*}=38.2845, \quad f_{5}^{*}=21.7155, \\
& f_{6}^{*}=60.0000, \quad f_{7}^{*}=38.7229, \quad f_{8}^{*}=21.2771, \quad f_{9}^{*}=60.0000, \quad f_{10}^{*}=42.1179,
\end{aligned}
$$




$$
f_{11}^{*}=17.8821, \quad f_{12}^{*}=60.0000, \quad f_{13}^{*}=36.1235, \quad f_{14}^{*}=23.8765
$$

As can be seen from the total link flows, over two thirds of the decision-makers chose to telecommute on the fourth day, with telecommuting being selected by more than half of the decision-makers on any given day. Hence, the number of commuters is lowest on day four and highest on day 5. 


\section{Summary and Conclusions}

In this paper, we have developed a multiperiod, multiclass, multicriteria network equilibrium model for the determination of telecommuting versus commuting decision-making over space and time. The model considers distinct classes of decision-makers, each of whom weights the three criteria of travel time, travel cost, and opportunity costs distinctly. The weights are not only class-dependent but also link-dependent. We conceptualize the problem, which assumes a finite time horizon, typically, a five day work week, through the use of a space-time network which abstracts the decision-making process as the selection of paths over the network. The paths consist of routes that are taken between residential and employment locations within each time period and also include connecting links which join subnetworks between successive periods.

We derive the equilibrium conditions and provide the finite-dimensional variational inequality formulation. We also provide qualitative properties of the solution pattern. We propose an algorithm for computational purposes and provide convergence results. Finally, we present numerical examples which illustrate the model.

This research is the first to allow for the prediction of how frequently decision-makers choose to telecommute versus to commute and the flow patterns that will prevail over space and time.

Future research will include empirical applications. 


\section{Acknowledgments}

The authors are grateful to the editor and to the two anonymous referees for their helpful comments and suggestions.

This research was supported by NSF Grant No. IIS-0002647. The first author's research was also supported by NSF Grant No. INT-0000309. This support is gratefully acknowledged. 


\section{References}

Albertson LA (1977) Telecommunication as a travel substitute: aome psychological, organizational, and social aspects. Journal of Communication 27: 32-43

Beckmann MJ, McGuire CB, Winsten CB (1956) Studies in the economics of transportation. Yale University Press, New Haven, Connecticut

Dafermos S (1981) A multicriteria route-mode choice traffic equilibrium model. Lefschetz Center for Dynamical Systems, Brown University, Providence, Rhode Island

Dafermos SC, Sparrow FT (1969) The traffic assignment problem for a general network. Journal of Research of the National Bureau of Standards 73B: 91-118

Dial RB (1979) A model and algorithms for multicriteria route-mode choice. Transportation Research 13B: 311-316

Dial R (1996) Bicriterion traffic assignment: basic theory and elementary algorithms. Transportation Science 30: 93-111

Dial RB (1999) Network-optimized road pricing: part I: a parable and a model. Operations Research 47: 54-64

Glater JD (2001) Telecommuting's big experiment. New York Times May 9: C1

Hafner K (2000) Working at home today? New York Times November 2: E7-E8

Harkness RC (1977) Technology assessment of telecommunications / transportation interactions. Stanford Research Institute, Menlo Park, California

International Telework Association \& Council (2000) Telework America (TWA) 2000 key findings.http://www.telecommute.org/twa/twa2000/research_results_key_shtml

Jones Jr DW (1973) Must we travel: the potential of communications as a substitute for urban travel. Report No. PB-227-185/6, Stanford University, Institute for Communications Research, Palo Alto, California 
Khan AM (1976) Travel vs. telecommunications: current understanding, part 1. Transportation Journal 10: 203-216

Kinderlehrer D, Stampacchia G (1980) An introduction to variational inequalities and their applications. Academic Press, New York

Korpelevich GM (1977) The extragradient method for finding saddle points and other problems. Matekon 13: 35-49

Leurent F (1993) Cost versus time equilibrium over a network. European Journal of Operations Research 71: 205-221

Leurent F (1996) The theory and practice of a dual criteria assignment model with continuously distributed values-of-times. In: Lesort JB (ed) Transportation and traffic theory, pp. 455-477. Pergamon, Exeter, England

Leurent F (1998) Multicriteria assignment modeling: making explicit the determinants of mode or path choice. In: Marcotte P, Nguyen S (eds) Equilibrium and advanced transportation modelling, pp. 153-174. Kluwer Academic Publishers, Boston, Massachusetts

Marcotte P (1998) Reformulations of a bicriterion equilibrium model. In: Fukushima M, Qi L (eds) Reformulation: nonsmooth, piecewise smooth, semismooth and smoothing methods, pp. 269-292. Kluwer Academic Publishers, Dordrecht, The Netherlands

Marcotte P, Nguyen S, Tanguay K (1996) Implementation of an efficient algorithm for the multiclass traffic assignment problem: In: Lesort JB (ed) Proceedings of the 13th international symposium on transportation and traffic theory, pp. 217-226. Pergamon Press, Exeter, England

Memmott III FW (1963) The substitutability of communications for transportation. Traffic Engineering 33: 20-25

Mokhtarian PL (1990) A typology of relationships between telecommunications and transportation. Transportation Research A 24: 231-242 
Mokhtarian PL (1991) Telecommuting and travel: state of the practice, state of the art. Transportation 18: 319-342

Mokhtarian PL (1998) A synthetic approach to estimating the impacts of telecommuting on travel. Urban Studies 35: 215-241

Mokhtarian PL, Handy SL, Salomon I (1995) Methodological issues in the estimation of travel, energy, and air quality impacts of telecommuting. Transportation Research $A$ 29: 283-302

Nagurney A (1999) Network economics: a variational inequality approach, second and revised edition. Kluwer Academic Publishers, Dordrecht, The Netherlands

Nagurney A (2000) A multiclass, multicriteria traffic network equilibrium model. Mathematical and Computer Modelling 32: 393-411

Nagurney A, Aronson J (1989) A general dynamic spatial price network equilibrium model with gains and losses. Networks 19: 751-769

Nagurney A, Dong J (2000) A multiclass, multicriteria traffic network equilibrium model with elastic demand. Transportation Research $B$, in press

Nagurney A, Dong J, Mokhtarian PL (2000) Integrated multicriteria network equilibrium models for commuting versus telecommuting. Isenberg School of Management, University of Massachusetts, Amherst, Massachusetts

Nagurney A, Dong J, Mokhtarian PL (2001) Multicriteria network equilibrium modeling with variable weights for decision-making in the Information Age with applications to telecommuting and teleshopping. To appear in Journal of Economic Dynamics and Control

Nagurney A, Kim DS (1991) Parallel computation of large-scale dynamic market network equilibria via time period decomposition. Mathematical and Computer Modelling 15: 55-67

Nilles JM (1988) Traffic reduction by telecommuting: A status review and selected bibliography. Transportation Research A 22: 301-317 
Nilles JM, Carlson Jr. FR, Gray P, Hanneman GJ (1976) The telecommunications - transportation tradeoff: options for tomorrow. John Wiley and Sons, Inc, New York

Quandt RE (1967) A probabilistic abstract mode model. In: Studies in travel demand VIII. Mathematica, Inc., Princeton, New Jersey, pp. 127-149

Salomon I (1986) Telecommunications and travel relationships: a review. Transportation Research A 20: 223-238

Schneider M (1968) Access and land development. In: Urban development models. Highway Research Board Special Report, 97: 164-177

Shore J (2000) Telework - the future is now. Office of Governmentwide Policy, U. S. General Services Administration, October 2000, http://www.pueblo.gsa.gov/telework.htm

Takayama T, Judge GG (1971) Spatial and temporal price and allocation models. NorthHolland, Amsterdam, The Netherlands 